\title{
QUELQUES JALONS POUR UNE HISTOIRE DES RELATIONS PUBLIQUES EN FRANCE, DES ANNÉES 1900 JUSQU'AU DÉBUT DES ANNÉES 80
}

\author{
Valérie Carayol, Bruno Chaudet \& Alexander Frame ${ }^{1}$
}

Le texte se propose, à partir d'une dizaine d'interviews, réalisés entre avril et septembre 2014 avec d'actuels et d'anciens responsables d'associations professionnelles de relations publiques, la consultation des archives de l'AFREP (Association française des relations publiques) et la lecture d'ouvrages scientifiques et professionnels comportant des éléments d'histoire de la profession, de recenser quelques jalons du développement de la profession en France et de soulever quelques questions auxquelles de futures études pourront répondre. Notre questionnement porte essentiellement sur la construction de la légitimité de la fonction, depuis les pionniers du début du siècle dernier jusqu'à la fin des années 70 , soit avant l'éclosion de la fonction communication à proprement parler et celle de la figure du DIRCOM. Il se veut une étape dans un champ en expansion en Europe, celui des recherches à caractère historique sur le développement des professions de communication et leurs pratiques.

1 Valérie Carayol est Professeure en Sciences de l'information et de la communication à l'Université Bordeaux Montaigne, et Directrice du laboratoire de recherche MICA (EA 4426). Bruno Chaudet est Maître de conférences à l'Université Rennes 2. Alexander Frame est Maître de conférences à l'Université de Bourgogne.

Recherches en communication, $\mathrm{n}^{\circ} 41$ (2014). 
Le terme de relations publiques reste en France attachéà un ensemble de pratiques communicationnelles des entreprises et des organisations relativement étroit, dont l'accroissement et le développement a donné lieu à une multiplication de termes : communication corporate, institutionnelle, organisationnelle, stratégique, etc.

Parler de relations publiques, en France, a un côté suranné et presque vieillot et fait souvent référence à un ensemble de pratiques professionnelles à caractère normatif, en usage jusque dans les années 80, mais aujourd'hui souvent dépassées (D'Almeida \& Carayol, 2014).

Le terme pourtant, a été utilisé de manière extensive par une communauté de professionnels de la communication français avant que son usage ne s'étiole et ne laisse la place à une autre terminologie, avec le développement de la profession et celui concomitant des formations universitaires et de la recherche. Aujourd'hui quasiment aucune formation universitaire française ne porte un intitulé comportant les termes relations publiques bien que le champ professionnel concerné fasse l'objet de très nombreuses formations et, depuis la fin des années 80 , d'un ensemble de recherches important sous l'étiquette « communication des organisations ». Le développement de la fonction communication dans les organisations a joué un rôle important dans la désaffection pour l'appellation « relations publiques », le terme n'étant plus assez représentatif, après les années 80 , de la multiplicité des pratiques mises en œuvre - des relations presse à la publicité - par les directions de la communication.

L'évolution historique du champ professionnel des relations publiques et de la communication des organisations a surtout été étudié dans l'espace francophone au Canada (Dumas, 2010 ; Maisonneuve, 2004) ou en Belgique (Gryspeerdt, 2004) mais n'a, jusque-là, pas fait l'objet de beaucoup de recherches en France, comme cela a déjà été souligné (Walter, 1995 ; Carayol, 2004, 2010), contrairement au champ du journalisme par exemple, dont l'évolution a été souvent scrutée et analysée (Delporte, 1995, 1999 ; Le Cam \& Ruellan, 2014).

L'intérêt pour le développement de recherches à caractère historique, qui s'est développée ces dix dernières années à l'échelle européenne (Rodriguez-Salcedo \& Watson, 2012), invite à se pencher sur le cas français, à partir de nouvelles sources.

La présente étude propose quelques éléments de repères sur l'apparition et le développement des relations publiques en France. Elle ne prétend pas faire le tour de la question mais propose, à partir d'une dizaine d'interviews, réalisés entre avril et septembre 2014 avec 
d'actuels et d'anciens responsables d'associations professionnelles de ce secteur, la consultation des archives de l'AFREP (Association française des relations publiques) et la lecture d'ouvrages scientifiques et professionnels comportant des éléments d'histoire de la profession, de recenser quelques jalons du développement de la profession. Notre questionnement porte essentiellement sur la construction de la légitimité de la fonction, depuis les pionniers du début du siècle dernier jusqu'à la fin des années 70, soit avant l'éclosion de la fonction communication, à proprement parler, et celle de la figure du DIRCOM, le directeur de la communication, qui aura par la suite vocation à rassembler sous sa responsabilité toutes les facettes des métiers de la communication de l'organisation, du marketing aux relations publiques.

Les relations publiques, en France, se sont essentiellement développées après la Seconde Guerre mondiale. Certes, il y a eu des opérations de relations publiques auparavant mais la fonction, elle, a été créée en France après la mise en place du plan Marshall et des échanges entre personnalités des mondes économiques français et américains. Ces rencontres vont être le terreau et le point de départ de l'importation massive de nombreuses techniques de management et de communication américaines dont les relations publiques comme fonction intégrée dans les organisations. Le début de la professionnalisation et de la construction de ce champ professionnel débute donc véritablement en France après la Seconde Guerre mondiale (Chouchan \& Flahault, 2011).

Si l'essor des associations professionnelles en relations publiques après la Seconde Guerre mondiale marque le début de la construction de la légitimité de la fonction, les pratiques de relations publiques ont évidemment commencé avant que le nom et la fonction ne soient explicitement créés dans les organigrammes.

Nous donnerons dans un premier temps une définition aux pratiques de relations publiques, puisqu'elles s'appelaient à l'origine comme cela, en les illustrant avec un cas emblématique de manière à montrer que ces pratiques existaient évidemment en France avant la Seconde Guerre mondiale. Nous détaillerons ensuite la mise en place des associations professionnelles et des débats visant à la construction de ce champ professionnel en France. Le tournant de la crise de 1968 nous permettra enfin de souligner le contexte social et historique dans lequel les services de communication sont apparus et se sont développés massivement. 


\section{Le début des relations publiques en France : des public relations aux relations publiques}

Avant que le nom et la fonction n'existent véritablement, quelles opérations de relations publiques pouvons-nous repérer et qualifier comme telles avant la Seconde Guerre mondiale en France ? Car comme le note évidemment C. Malaval (1996), « Les préoccupations communicationnelles existaient dans l'entreprise bien avant la deuxième guerre mondiale ».

Comme le souligne Jean-Pierre Beaudoin ${ }^{1}$, Michelin est probablement l'une des premières grandes organisations à développer une politique de relations publiques. Ainsi, l'entreprise fournit des pneus à des coureurs cyclistes dès 1898. Elle édite également un Guide Rouge en 1900 destiné aux professionnels et plus précisément aux routiers. Elle poursuit dans sa lancée en créant en 1910 les premières bornes de signalisation d'entrée dans les communes puis, en 1918, installe des bornes d'indication de direction et de kilométrage. C'est une politique de relations publiques au sens où Michelin prend en compte les intérêts de ses publics et met en place un dispositif qui leur facilite l'existence.

Michelin est une entreprise qui a compris quasiment depuis son origine (1889), que c'est en déployant tous les instruments de communication utiles à ceux qui allaient acheter ses pneus qu'ils allaient installer leur marque sur leur territoire. Alors ils ont fait des cartes, des guides, des bornes et c'est Michelin. Et on occupe un territoire de marque avec des moyens de communication (Entretien avec Jean-Pierre Beaudoin, 26 mai 2014).

Nous avons donc là une démarche qui se veut rigoureuse et qui illustre la conception des relations publiques qui prévalait à ce moment là. Michelin est une entreprise qui répond à la question : Qui sont mes publics ? Réponse : Les gens qui circulent en automobile. Comment puis-je construire des relations avec ces publics ? Avec tous les instruments qui vont leur rendre des services. L'organisation travaille à augmenter sa légitimité en soulignant les contributions qu'elle apporte

1 Entretien avec Jean-Pierre Beaudoin, 26 mai 2014. Jean-Pierre Beaudoin est un dirigeant d'entreprise, consultant en communication, essayiste et professeur en sciences de la communication (Wikipédia). 
au corps social ; elle rend des services, met en avant sa responsabilité sociale (Arzeno-Martin, 1993) et le fait savoir grâce à des pratiques qui préfigurent celles des relations publiques à venir.

La création de la fonction Relations Publiques dans les organisations semble pouvoir remonter à 1947. Cette année-là, François LuléDejardin (Shell France), Lucien Matrat (Caltex) et Jean Choppin de Janvry (Esso), partent en mission de productivité aux Etats-Unis où ils découvrent les public relations. « De retour en France, ils feront figure de précurseurs, en mettant en place des services de relations publiques dans leur société respective »(Viale, 1997, p. 39). De nombreuses compagnies, dont en premier lieu les filiales françaises de compagnies pétrolières anglo-saxonnes, vont donc envoyer des hommes participer à des missions de productivité et revenir avec l'idée de développer les relations publiques telles qu'ils ont pu les observer aux Etats-Unis, terre d'invention au début du XX ${ }^{\text {ème }}$ siècle de cette méthode d'ingénierie du consentement comme l'a appelée Edward Bernays (1947) ou, dans une version plus politiquement correcte avec Ivy Lee, une méthode qui consiste à expliquer à ses publics quel est le rôle des entreprises et leur utilité dans la communauté locale, régionale et nationale. Il s'agit alors « pour les professionnels des relations publiques de modifier la représentation que se fait le public de l'industrie pétrolière en fournissant des explications objectives principalement en direction des relais d'opinion » (Viale, 1997, p. 59). Esso organise alors des réunions d'information, édite la revue Pétrole et Progrès, propose des ouvrages de vulgarisation aux enseignants...

Les importateurs de la fonction relations publiques en France l'intègrent exactement dans le même esprit que Michelin lorsqu'il l'a développée. Ils l'importent parce qu'il y a un intérêt pour l'entreprise à construire une relation avec certaines catégories de publics. Il y a donc une analyse des publics qui ne se réduit pas aux clients du point de vue du marché. «On inscrit le marché dans la société et on regarde ce que sont les besoins des publics qui nous concernent dans leur fonctionnement social et pas seulement dans leur fonctionnement de marché ${ }^{1} \gg$. Mais pourquoi les grandes entreprises pétrolières entreprennent-elles les premières des programmes de relation avec l'enseignement?

Parce qu'il y a la crise du pétrole au Proche-Orient, la nationalisation du sous-sol par les pays du Golfe...

1 Entretien avec Jean-Pierre Beaudoin, 26 mai 2014. 
A l'époque, en France, c'était la lutte contre l'oligopole anglo-américain du pétrolequiétaitconsidérépolitiquement comme scandaleux parce que dans le fond il suffisait de faire un trou dans le sol pour avoir du pétrole et le vendre très cher à l'autre bout. C'est cette vision réductionniste de la critique politique de l'industrie pétrolière qui a conduit les entreprises de ce secteur à développer des programmes pédagogiques pour expliquer ce qu'est le pétrole. Et ils commencent à l'expliquer de l'exploration jusqu'à la pompe, en montrant tous les métiers, tous les investissements, toutes les transformations... et tous les bienfaits dont naturellement le plastique. Et ce sont les premiers programmes pédagogiques d'entreprise (Entretien avec Jean-Pierre Beaudoin, 26 mai 2014).

Les entreprises de distribution d'eau répliqueront ce modèle dans les années 1990 au moment où la critique politique et sociale de ce métier montera en puissance en avançant les mêmes arguments : « C'est scandaleux ces gens qui font du bénéfice sur l'eau. C'est un bien naturel, qui est dans l'environnement... » Le même schéma se répète donc, dans lequel nous retrouvons les mêmes programmes transposés quelques décennies plus tard. En somme, ce sont les périodes de crise qui vont donner à la fonction relations publiques ses périodes de développement, qui semblent résulter d'une approche défensive, comme nous le verrons avec la crise de 1968 .

Dans les organisations publiques, les services de relations publiques se développent en France dès le début des années 50. Un service important est créé au service des Postes et Télécommunications en 1952 - qui occupera par la suite entre 150 et 200 personnes - puis vers 1958 dans les autres ministères.

\section{La construction d'une légitimité}

$\mathrm{Du}$ point de vue de la représentation de la fonction et de la construction de sa légitimité, il faut retenir la date du 27 décembre 1950 à laquelle les pionniers (Jean Choppin de Janvry, Lucien Matrat) créent la première association professionnelle de relations publiques en France sous le nom de Club de la Maison de Verre. Le nom faisait alors clairement référence aux principes d'Ivy Lee : la transparence, 
dire toute la vérité. Le but du club est alors de développer les pratiques ainsi qu'une théorie des relations publiques en France.

Les premières agences voient le jour : Information \& Entreprise de Jacques Coup de Fréjac, Relations d'Henri Pineau et Géo-Charles Véran, Orel de Georges Serrel.

L'association se met rapidement d'accord pour employer le terme de « relations publiques » pour désigner ce nouveau métier en imitation des «public relations » américaines. Mais la traduction est, pour certains, fâcheuse car les relations publiques n'auraient absolument pas le même sens que public relations.

Le mot public en anglais ne veut pas dire public en français, il veut dire privé. Qu'est-ce qu'une public school ? C'est une école privée ? Qu'est-ce qu'une public company ? Ce n'est pas une société publique. C'est une société qui appartient à ses publics, c'est-à-dire à des individus (Entretien avec Jean-Baptiste de Bellescize, Paris, 6 mai 2014).

L'expression « relations publiques » ne traduit pas ce phénomène. Cela pourrait expliquer pourquoi Thierry Wellhoff, actuel président du Syntec RP, a fait récemment évoluer l'adjectif " publiques » en « publics » pour signifier relations avec les publics. Pour certains, c'est cette mauvaise traduction originelle qui se serait avérée inadéquate et aurait généré en France toute une série de mauvaises perceptions sur ce métier. Cette erreur de traduction aurait eu une incidence forte sur la perception des relations publiques en France.

Au-delà des problèmes sémantiques, les professionnels se sont surtout attachés dans les premiers temps à légitimer leur profession. Très rapidement, il s'est agi de construire des frontières très strictes entre le journalisme, la publicité commerciale et les relations publiques. Tous les efforts étaient tendus vers la volonté de se démarquer de la propagande. Il est vrai que les pionniers des relations publiques aux Etats-Unis utilisaient des techniques qui s'inspiraient largement de la propagande ${ }^{1}$ et tout semblait se passer comme si les Français voulaient

1 On peut citer les pratiques de la commission Creel pour persuader les américains d'entrer dans la Première guerre mondiale. Et bien sûr Edward Bernays, issu de la commission Creel, célèbre pour son livre : (1928). Propaganda, comment manipuler l'opinion publique en démocratie 
rompre avec ce modèle, qui faisait écho à la communication de guerre et notamment à la propagande des vaincus ${ }^{1}$.

Dans ce contexte déontologique et identitaire particulier, l'Aprorep (Association des professionnels des relations publiques) est ainsi créée en 1952 par quelques membres du Club de la Maison de Verre qui souhaitent développer une ligne de défense des relations publiques avec un code déontologique contre certaines pratiques de professionnels qui se prévalaient du terme de relations publiques. Leurs efforts se traduisent en 1954 par la création d'un code professionnel des relations publiques. Ce code a alors pour fonction d'apporter des règles et de préciser les finalités du métier de relations publiques. On y lit que :

La spécialisation des fonctions et des activités imposées par le progrès technique a élevé des cloisons entre les groupes qui composent la Société, et entre les individus appartenant à ces groupes. Il en résulte une insuffisance des communications et une absence de connaissance mutuelle pouvant aller jusqu'à une rupture complète des relations (...). On appelle Relations Publiques les activités déployées par un groupe en vue d'établir et de maintenir de bonnes relations entre les membres du groupe et entre le groupe et les différents secteurs de l'opinion publique (Walter, p. 35).

Les principes des relations publiques se développent alors de pair avec une critique de la société industrielle et l'idée selon laquelle ce sont les relations qui vont permettre de résoudre problèmes et antagonismes sociaux. Il s'agit de développer des relations avec son environnement au sens large : "Personnels, actionnaires, agents de distribution, clients et fournisseurs, enseignement, presse, organismes syndicaux, administrations publiques, institutions législatives et exécutives, etc. » (op. cit.) Mais cette dimension globale, comme on le verra plus loin, ne sera pas perçue immédiatement par les chefs d'entreprise qui attendront en fait les grands conflits sociaux de la fin des années 60 pour commencer à prendre en compte l'environnement et donc les relations

1 Il est à noter que les premiers relationnistes français comme Jacques Coup de Fréjac par exemple étaient issus des bureaux d'information des maréchaux français pendant la Deuxième Guerre mondiale qui avaient connu des pratiques de propagande et avaient pris conscience que le moral des troupes en arrière du front était très important pour gagner la guerre. 
avec leurs publics. Ce code, créé par l'Aprorep, sera ensuite remplacé par le code d'Athènes en 1965, sous-titré « Code d'éthique international des relations publiques $\gg{ }^{1}$.

Le Club de la Maison de Verre et l'Aprorep décident de fusionner en 1955 et de créer L'AFREP (Association française des relations publiques). Suit alors une période de création d'associations professionnelles qui marque tout autant la volonté que la difficulté de s'entendre sur ce que doivent être les relations publiques.

En 1955, Géo-Charles Véran crée le Syndicat national des agents de relations publiques. La même année, Jacques Coup de Fréjac et Georges Serell fondent le Syndicat national des conseils en relations publiques (SNCRP). L'année suivante, André Hurtrel crée l'Union nationale des attachés de presse (UNAP). Philippe Boiry évoque cette époque en soulignant que l'important était de faire masse.

Afin de regrouper le plus grand nombre de personnes, il était créé une catégorie de membres associés, qui payaient une cotisation relativement plus élevée. En faisaient partie, par exemple, le futur maire du $8^{\text {ème }}$ arrondissement, chômeur à cette époque, qui n'avait rien à voir avec les $\mathrm{RP}$, un ancien combattant méritoire, qui ne connaissait rien aux RP... Philippe fit remarquer à Hurtrel « qu'une association dite de pêcheurs à la ligne qui comportait plus de joueurs de boules que de pêcheurs n'en était pas une », ce dernier répondit que ce qui comptait c'était le nombre, faire nombre ! Et bien sûr financièrement ! (Entretien avec Philippe Boiry réalisé par Janine Aubouy Dutreix).

En 1958 ce sera au tour d'une chambre syndicale des relations publiques. Le Synap (Syndicat national des attachés de presse) sera créé en 1960 à l'initiative de Philippe Boiry qui voulait créer un syndicat ne comportant que des attachés de presse professionnels contrairement à l'UNAP. « Pour montrer qu'il n'y avait pas de scission avec l'UNAP, il demanda à deux vice-présidents de l'UNAP, Jacques Morlaine et Pierre Violet, parmi les fondateurs de l'UNAP, d'être les deux vice-présidents du SYNAP². »

1 http://www.infopressecom.org/cms/page/code-dathenes, consulté le 18 mai 2014. Le Code d'Athène a été adopté par le Centre Européen des Relations Publiques.

2 Entretien avec Philippe Boiry réalisé par Janine Aubouy Dutreix 
L'enjeu était de délimiter un territoire professionnel et de construire des principes déontologiques qui marquent une différence nette entre publicité et relations publiques, propagande et relations publiques.

Cette recherche de fondements déontologiques se cristallisera dans l'arrêté Peyrefitte de 1964, du nom du ministre de l'information de l'époque, qui propose une distinction entre propagande et relations publiques dans son article premier.

Le SNCRP a fait une chose majeure. Il a négocié, en 1964, avec le ministre de l'information d'alors qui était Alain Peyrefitte, un arrêté ministériel qui a été l'arrêté qui a défini la profession. Cet arrêté Peyrefitte est très intéressant parce qu'il structure les métiers de la communication autour de trois métiers incompatibles : le journalisme, la publicité et les relations presse. Il structure ces trois métiers comme dans les métiers de droit. On ne peut pas être avocat, procureur et juge en même temps. On ne peut pas être journaliste, publicitaire et homme de relations de presse en même temps. (Entretien avec Jean-Baptiste de Bellescize, Paris, 6 mai 2014).

Mais la profession se trouvait dispersée : outre l'UNAP et le SYNAP, il y avait l'UJJEF, Union des Journaux et Journalistes d'Entreprise de France, l'AFREP et le SNCRP.

En 1966, les 6 et 7 mai, Franck Bauer, président de l'AFREP proposa que ces cinq groupements qui représentaient la profession se réunissent à Strasbourg pour créer une sorte de comité informel, un comité de liaison des présidents, composé de Franck Bauer, initiateur, Robert Cusin, Henri Dumon, Paul Aubé et Philippe A. Boiry, qui déboucha cinq ans plus tard sur la création de la Fédération Française des Relations Publiques, FFRP (1971). Cette Fédération travailla énormément, créant un code de déontologie commun à tous les groupements. La présidence fut confiée chaque année au président de l'un des groupements (elle fut dissoute en 1996), car le président de l'UNAP d'alors s'en était totalement désintéressée lorsque ce fut le tour de son association. (Entretien avec Philippe Boiry réalisé par Janine Aubouy Dutreix). 
A cette époque, la question de la distinction entre journalisme, publicité et relations publiques était toujours d'actualité. Ainsi peut-on lire l'éditorial de Jacques Coup de Fréjac dans les nouvelles brèves de l'Afrep :

Pendant 1'adolescence des relations publiques en France, certains responsables des secteurs publics et privés, voire certains professionnels, ont pu se tromper de bonne foi sur les limites respectives de la publicité, des relations publiques et de la presse. Aujourd'hui ceux qui continuent à le faire sont de mauvaise foi et leur comportement est d'autant plus inexcusable qu'il cache des fins intéressées. Entre territoires voisins, il existe des frontières naturelles ou réglementaires. Il existe aussi parfois des zones de « no man's land ». L'habitude veut que ce soit le terrain d'action des contrebandiers. Il n'y a pas de place dans notre profession pour ces clandestins ${ }^{1}$.

Et de poursuivre qu'il faut absolument distinguer les relations publiques, de la publicité commerciale, de la publicité financière et de la publicité institutionnelle.

Nous connaissons l'utilité de la publicité institutionnelle mais nous regrettons son appellation qui entretient une confusion. Cette forme de promotion doit être conçue par le responsable des relations publiques qui participe à la définition de la politique à long terme de l'entreprise ${ }^{2}$.

C'est la guerre des territoires légitimes entre les métiers.

\section{Les relations publiques après la crise de 1968}

Si la création de ces associations professionnelles a indéniablement joué un rôle moteur dans le développement et la légitimité de ce métier, les chefs d'entreprise vont véritablement saisir l'enjeu et l'importance de cette fonction avec la crise de 1968. Nous avons en effet vu que les relations publiques prônaient le dialogue et les relations avec l'ensemble des publics. Or, la culture patronale française dans les années 60 n'était

1 (1972) Nouvelles brèves de l'association française des relations publiques, 76.

2 (1972) Nouvelles brèves de l'association française des relations publiques, 76. 
pas du tout tournée vers le dialogue, qu'il soit interne ou externe. «En France, les plus longs à comprendre le rôle des attachés de presse furent les chefs d'entreprise ${ }^{1} »$.

En ce qui concerne les relations internes, les patrons n'avaient pas à cette époque la visée humaniste que souhaitaient promouvoir les précurseurs des relations publiques. La mise en place du salaire minimum interprofessionnel garanti (SMIG) en 1950 est à cet égard particulièrement éclairante. Le CNPF (Conseil national du patronat français) s'est en effet toujours opposé à la mise en place du SMIG. C'est l'Etat qui a du l'imposer dans des conditions absolument hallucinantes. Henri Weber raconte dans Le Parti des patrons : Le CNPF, 1946-1990, les conditions de cette négociation entre l'Etat et le CNPF. Il a en effet d'abord fallu s'entendre sur ce que l'on pouvait entendre par salaire minimum vital :

Alors on évaluait le budget calorique minimum. Mais il y avait toujours un type dans la sous-commission alimentaire pour affirmer : « Mais non, on peut vivre avec 100 calories de moins ! ». C'était affreux. L'évaluation du budget non alimentaire prêtait davantage encore à contestation. Là aussi, à chaque fois, vous aviez un type qui disait que l'on pouvait habiller quelqu'un correctement pour moins que ça. Et ils ont fait, au CNPF, dans la salle des négociations, une exposition de la garde-robe du Smigard. Il y avait le costume noir qui devait servir pour le mariage, les jours de fête et l'enterrement. Il y avait des chemises, des chaussures. (Weber, 1991).

Voilà l'état des relations humaines dans l'entreprise, bien loin des propositions des associations professionnelles. C'était l'époque du taylorisme triomphant. La communication et les relations publiques internes n'avaient absolument aucune place dans l'entreprise. Mais le taylorisme commence à être critiqué et plusieurs rapports vont souligner l'importance de la prise en compte les relations humaines dans l'entreprise. En 1963, François Bloch-Lainé, haut fonctionnaire, encourage par exemple le développement de ces dernières dans l'entreprise dans un livre à succès intitulé Pour une réforme de l'entreprise (1963). En 1972, le Centre des Jeunes Dirigeants

1 Entretien avec Philippe Boiry réalisé par Janine Aubouy Dutreix. 
d'entreprise publie sur le même thème L'Information dans l'entreprise. Le 13 février 1975, Pierre Sudreau remet un rapport à Valéry Giscard d'Estaing, alors Président de la République, intitulé La Réforme de l'entreprise.

Mais c'est véritablement le choc des évènements de 1968 qui va réveiller les patrons mais aussi les administrations publiques. C'est 1968 qui va les convertir à la nécessité de gérer les relations avec les publics. Il y aura alors la création de services appelés d'InformationCommunication puis de directions de la communication qui manifestent une prise de conscience de la fonction stratégique de la communication pour les organisations : Saint-Gobain en 1970, L'Oréal en 1973, RhônePoulenc en 1977... (Heurtebise, 1989).

C'est à cette même époque, que la mission de Jacques Baumel, secrétaire d'Etat chargé des relations publiques (1969-1972), auprès du Premier Ministre Chaban-Delmas, contribue à légitimer la profession, en lui donnant une visibilité au sommet de l'état, ce qui a contribué à légitimer fortement les pratiques de relations publiques.

On peut s'attarder sur la création du service communication du CNPF en 1970 créé par Michel Frois, transfuge du SIRPA (Service d'information et de relations publiques des armées). Ce qui arrive au CNPF, dans le fond, est révélateur de la prise de conscience de l'importance de l'information, surtout pour développer une ligne de défense après 68. En fait, le CNPF n'avait pas vu venir la grève générale de mai-juin 1968 et c'est à cette époque qu'ont lieu les premières séquestrations de patrons. Henri Weber écrit :

Les patrons ne sont pas bavards sur cette période. Ils parlent avec beaucoup de pudeur de la grève dans leurs usines. « Oui, on était occupé. Un tel était séquestré. Pas longtemps, notez bien. En gros, ça ne se passait pas trop mal. » Mais, sur le moment, ils ont vécu les événements comme un violent traumatisme. C'est à partir de ce moment-là que le CNPF va faire sa révolution médiatique, suivie par un grand nombre d'entreprises. L'innovation institutionnelle de loin la plus lourde de conséquences, sur le moment passé inaperçue, est la création d'une troisième grande commission du CNPF : la direction générale de l'information. 
Les créations des directions de communication datent de cette période et sont le produit de la jonction de la communication interne, qui s'était développée au sein des services des relations humaines, et de la communication externe qui était le produit direct des relations publiques.

Lors de ses assises nationales de 1972, le CNPF inscrit alors « La croissance, l'entreprise et les hommes » au centre de ses débats et décide d'engager une série d'actions en direction des médias en invitant 175 journalistes de la presse écrite, parlée et télévisée ${ }^{1}$. Deux grands thèmes de travail sont alors débattus : Croissance et société et L'homme dans l'entreprise au sein desquels la question de la communication est centrale. On y parle d'esprit de communication, d'ouvriers de plus en plus qualifiés qui ont besoin d'être informés... Il s'agit de convertir les chefs d'entreprise à la communication.

Prononcé lors de ces assises, le discours d'Antoine Riboud (PDG de Danone) marque un tournant. Connu aujourd'hui sous le nom de discours de Marseille, il prend acte des évolutions majeures de la société française et appelle les chefs d'entreprise à mettre en place des systèmes d'information qui impliquent le personnel. Il appelle à dépasser le strict point de vue économique pour prendre en compte d'autres dimensions de la personne humaine comme la solidarité, la responsabilité et la personnalisation. Antoine Riboud souhaite également élargir les responsabilités de l'entreprise au-delà des murs de l'usine. L'entreprise a désormais des responsabilités vis-à-vis de la collectivité tout entière.

Jacques Coup de Fréjac en fera son édito en décembre 1972 dans la revue La Maison de Verre ${ }^{2}$ :

Le CNPF nous a invité - implicitement, si ce n'est explicitement - à orienter nos services dans cette direction. Si les choses se font sans nous, nous serons les seuls à blâmer. Pour qu'elles se fassent avec nous et même par nous, nous n'avons pas d'autres choix que d'assumer la « globalité » de la fonction de communication ${ }^{3}$.

La fonction communication en lieu et place des relations publiques apparait pour la première fois.

1 (1972). La Maison de Verre, 80.

2 Un titre inspiré du Club antérieur du même nom tout autant que des principes de transparence prônés entre autres par Ivy Lee.

3 (1972). La Maison de Verre, 80. 
Le rôle de l'information et donc des relations publiques internes aussi bien qu'externes prend alors de l'ampleur. Une nouvelle impulsion viendra de l'Etat en la personne de Valéry Giscard d'Estaing. Pendant sa Présidence, il crée la direction générale pour les Relations avec les publics en 1977 (Delorme, 2000).

La grande diffusion des relations publiques dans l'administration publique, c'est Giscard. Lorsqu'il crée la direction générale pour les Relations avec les publics, ça veut dire que vous en créez dans 101 départements. Tout d'un coup vous changez d'échelle. Dans cette période-là, la profession dans le secteur public devient plus nombreuse que dans le secteur privé. Le public a joué un rôle d'accélérateur (Entretien avec Jean-Pierre Beaudoin, 26 mai 2014).

\section{Les tendances de fond et l'espace public}

D'un point de vue plus sociologique, et comme le résume Jean-Pierre Beaudoin, il y a trois grands changements qui vont créer les conditions pour que la fonction s'installe et se développe en France.

Il y a d'abord l'évolution démographique. La majorité de la population sort de l'enseignement secondaire et c'est l'époque où $50 \%$ de la France a son bac.

C'est un changement radical. La France devient intelligente. Ce n'est pas qu'elle était bête avant mais la France se reconnaît un niveau intellectuel capable de juger. A partir de là, les gens prennent la parole et les médias créent des rubriques qui donnent la parole aux gens. C'est la création d'un espace public dans lequel les gens considèrent qu'ils ont quelque chose d'intéressant à dire et les médias reconnaissent cette parole en la publiant.

C'est aussi la période où apparaissent les premiers grands magazines sur l'entreprise : L'Expansion (1967), Le Nouvel Economiste (1976),... Les gens veulent comprendre l'économie, l'entreprise, là où ils travaillent. Et c'est un grand succès. L'Expansion à l'époque est une référence dans le paysage. 
A partir du moment où vous mettez dans le paysage des médias qui parlent de l'entreprise et que les gens lisent, en face du patron, il y a des gens qui non seulement se considèrent intelligents mais qui se considèrent comme informés donc compétents (Entretien avec Jean-Pierre Beaudoin, 26 mai 2014).

Le troisième changement c'est l'évolution du droit avec le rapport Sudreau (1975) sur l'état des relations entre les salariés et les patrons dans l'entreprise. Ce rapport fait évoluer les choses significativement. C'est le premier rapport qui rend compte de la question de la communication interne dans l'entreprise et qui fonde la légitimité de la décision par le processus mis en place. C'est aussi un moment de la société que décrit bien Habermas (1997) et qu'il convient d'appeler l'avènement de la légitimité procédurale. C'est le fait que ce qui fonde la légitimité d'une décision, c'est le processus d'élaboration de la décision. Le rapport Sudreau préconise ce processus en interne puisque toute la réforme des relations entre employeurs et représentants de salariés, c'est la surcharge procédurale de toute décision avec les procédures de consultation des salariés.

Le terrain est désormais propice au développement rapide des pratiques professionnelles des relations publiques et le début des années 80 voit une extension considérable du nombre des professionnels du secteur. L'élection de François Mitterrand en 1982 et le développement, qui a immédiatement suivi, de la logique de projet prônée par Michel Rocard dans les administrations et les corps de l'état engendrera un besoin d'expertise en communication publique concomitant au besoin d'expertise des entreprises privées. Le responsable des relations publiques, dans toutes les sphères organisationnelles, publiques, comme privées, cède la place au DIRCOM (Walter, 1995) à cette même période. L'usage du terme de relations publiques s'étiole pour n'être quasiment plus utilisé à cette même période. 


\section{Références}

Entretiens réalisés avec Jean Amyot d'Inville, Jean-Pierre Beaudoin, Annie Blin, Philippe Boiry, Lionel Chouchan, Anne-Marie Cotton, Jean-Baptiste de Bellescize, Jean-François Flahault, Frédérique Pusey, Thierry Wellhoff.

Arzeno-Martin, H. (1993). Un retour vers le futur avec Lucien Matrat, le père fondateur des Relations Publiques Européennes. Communication et organisation, 4.

Bernays, E. (1947). The Engineering of Consent. The Annals of the American Academy of Political and Social Science, 250(1), 113-120.

Bernays, E. (2007). Propaganda, comment manipuler l'opinion en démocratie. Paris : Zones.

Bloch-Lainé, F. (1963). Pour une réforme de l'entreprise. Paris : Seuil.

Carayol, V. (2010). PR professionals in France: an overview of the sector. Journal of Communication Management, 14(2).

Carayol, V. (2004). France. Dans B. Van Ruler \& D. Vercic (Éd.). Public Relations and Communication management in Europe. A nation-by-nation introduction to Public Relations Theory and Practice (pp. 135-152). Berlin ; New York : Mouton De Gruyter.

Centre des jeunes dirigeants d'entreprise. (1972). L'Information dans l'entreprise.

Chouchan, L., \& Flahault, J.-F. (2011). Les relations publiques. Paris : PUF.

D'Almeida, N., \& Carayol, V. (2014). La communication organisationnelle, une question de communauté. RFSIC, 4.

Delorme, G. (2000). De Rivoli à Bercy : souvenirs d'un inspecteur des finances, 19521998. Paris : Comité pour l'histoire économique et financière de la France.

Delporte, C. (1995). Histoire du journalisme et des journalistes en France : (du XVIIe siècle à nos jours.) Paris : PUF.

Delporte, C. (1999). Les journalistes en France : 1880-1950. Paris : Seuil.

Dumas, M. (2010). Les relations publiques, une profession en devenir. Québec : PUQ.

Floris, B. (1996). La communication managériale. La modernisation symbolique des entreprises. Grenoble : PUG.

Gryspeerdt, A. (2004). Relations publiques et recherche en communication. Hermès, 38.

Habermas, J. (1997). Sur le droit et la démocratie. Note pour un débat. Le Débat, 97(5), 42-47.

Heurtebise, C. (1989). Dircoms : 10 ans après, l'heure du bilan. CB News, 136.

Landrin, X. Propagande, Encyclopcedia Universalis, consulté le 10 avril 2014. Disponible à : http://www.universalis-edu.com/encyclopedie/propagande/

Le Cam, F. , Ruellan, D., Adghirni, Z.L., Moura, D.O., \& Pereira, F.H. (Dir.). (2014). Changements et permanences du journalisme, coll. Communication et civilisation. Paris : L'Harmattan.

Le Moënne, C. (1994). Communication by smiling around et crise managériale. Réseaux, 64.

Maisonneuve, D. (2004). Les relations publiques: le syndrome de la cage de Faraday. Québec : PUQ.

Malaval, C. (1996). L'histoire des entreprises à travers sa presse. Entreprises et Histoire, $11,49-60$. 
Okay, A., Carayol, V., \& Tench, R. (Dir.). (2013). Researching the Changing Profession of Public Relations. Bruxelles : Peter Lang

Rodríguez-Salcedo, N. (2012). Mapping Public Relations in Europe: Writing National Histories against the US Paradigm. Disponible à : http://dadun.unav.edu/ handle/10171/27966

Viale, T. (1997). La communication d'entreprise: pour une histoire des métiers et des écoles. Paris : L'Harmattan.

Walter, J. (1995). Directeur de communication, les avatars d'un modèle professionnel. Paris : L'Harmattan.

Watson, T. (2012). Let's get dangerous: A Review of Current Public Relations Historical Scholarship. Dans PR and the Past in Local and International Contexts, Conférence 13 Novembre 2012, University of Technology Sydney (UTS), Sydney, Australia.

Watson, T., \& Likely, F. (2013). Measuring the edifice: Public relations measurement and evaluation practices over the course of 40 years. Disponible à : http://eprints. bournemouth.ac.uk/20494/

Weber, H. (1991). Le parti des patrons : Le CNPF (1946-1990). Paris : Seuil. 